\title{
ONE STROKE CURSIVE CHARACTER RECOGNITION USING COMBINATION OF DIRECTIONAL AND POSITIONAL FEATURES
}

\author{
Teng Long ${ }^{1}$, Lian-Wen Jin ${ }^{1}$, Li-Xin Zhen ${ }^{2}$, Jian-Cheng Huang ${ }^{2}$ \\ ${ }^{1}$ School of Electronics and Information \\ ${ }^{1}$ South China University of Technology, Guangzhou 510641, P.R.China \\ ${ }^{2}$ Motorola China Research Center, Shanghai, 200002, P.R.China
}

\begin{abstract}
This paper proposes a new hybrid approach of directional and positional features for on-line one stroke cursive character recognition based on Dynamic Time Warping (DTW) algorithm. In our camera based user interface, user inputs various kinds of characters including Chinese characters by moving fingertip. All strokes of the character are connected, so our recognizer is designed for one stroke cursive character recognition. A quadratic curve equation for local distance measure is employed in DTW to improve the robustness of the classifier especially for complicated characters. By reconstructing positional feature from directional feature, only directional vectors are needed to be recorded. Thus the template file size can be reduced a lot. As the template size is small (about $300 \mathrm{~K}$ including Chinese characters) and the templates can be easily customized by user, the recognizer is suitable for hand-held devices. The efficiency of our approach is demonstrated by the promising experimental results.
\end{abstract}

\section{INTRODUCTION}

On-line handwriting character recognition has been researched for more than 40 years. It provides a natural and convenient way for human-machine interaction. By employing a certain kind of on-line handwriting recognition technology, a handwriting input device, such as personal digital assistant (PDA), electronic tablet, or camera, can record and then convert the trajectories of a pen tip or a fingertip to machine characters when people writing on them. Compared with off-line character recognition, on-line character recognition can provide good interaction and adaptation capability because the writer can correct errors at real time and can customize his own writing style. The state of the art of on-line handwriting recognition methods and the problem field in general is given in a survey [1], and the state of the art of on-line Chinese character recognition is described in a recent paper [2].
In this paper, we propose an on-line one stroke cursive character recognition method using combination of directional and positional features. There are several papers [3-5] described their recognition method based on Dynamic Time Warping (DTW) algorithm [7]. They used different feature extractions and dissimilarity measures. Both of them can affect the accuracy of the recognition results directly. In this paper, the experimental results show the importance of the feature extraction and the efficiency when directional and positional feature are combined using a selecting strategy. In order to be applied in our camera based user interface, the classifier is designed for one stroke characters. Each Chinese or English character's strokes are connected when performing the stroke correspondence matching. Due to one cursive stroke but not several strokes correspondence matching, the recognizer is stroke number free and can recognize some terribly distorted characters even some strokes omitted. It is partly because that the recognizer is not affected by errors of the classification of separated strokes. In addition, the template size is small owes to two reasons: only directional vectors are recorded and our classifier can provide good classification ability with comparatively small number of templates.

\section{OUR CAMERA BASED USER INTERFACE}

In our camera based user interface, user inputs various kinds of characters including Chinese characters by moving fingertip.

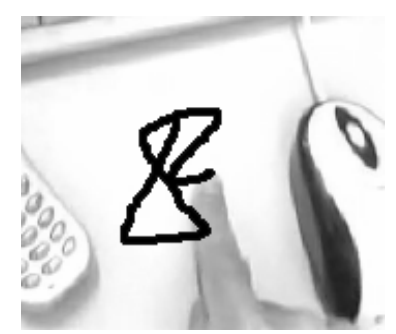

Fig. 1. A typical one stroke character "E" written by user's fingertip

*Project sponsored by: Motorola Human Interface Lab Research Foundation (No.D84110), NSFC (No.60275005), GDNSF (No.2003C50101, 04105938). 
The system can figure out the location of user's fingertip when it is moving. By some special actions defined as start and end points, user can write one stroke characters as the system records the trajectories of the user's fingertip by connecting each fingertip point recognized in each frame. A typical one stroke character "E" written by user's fingertip is shown in Fig. 1.

\section{FEATURE SELECTION AND EXTRACTION}

We choose the writing direction as the main feature of strokes of handwritten characters. When constructing templates for handwritten characters, only directional vectors are needed to be recorded. The positional feature vectors are dynamically regenerated from the directional vectors at run-time.

\subsection{Normalized discrete directional feature (NDDF)}

In our recognition system, templates are presented by strings of normalized discrete directional angle values. The angle value is demonstrated in Fig.2(a). It stands for the writing direction at an extracted feature point of a handwritten character's stroke. The value is an integer ranged from 0 to 255 linearly mapped from $0^{\circ}$ to $359^{\circ}$ for consideration of precision and storage problem. In section 5 , the experimental results show higher precision angle value can provide higher recognition rate. But this depends on sampling distance of feature points. We use a constant $\mathrm{d}$ for sampling distance, which is shown in Fig.2(b).

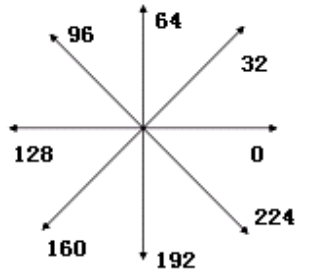

(a)

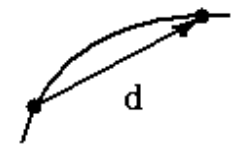

(b)
Fig. 2. (a) Normalized discrete directional angle value. (b) Two successive feature points extracted along the stroke's curve

\subsection{Sampling positional feature based on NDDF}

There is no positional feature stored in our templates. But as the sampling distance is a constant, we can reconstruct the characters' figure in templates by:

$$
\begin{aligned}
& x_{i+1}=x_{i}+d * \cos \left(\theta_{i} / 256 * 2 \pi\right) \\
& y_{i+1}=y_{i}+d * \sin \left(\theta_{i} / 256 * 2 \pi\right)
\end{aligned}
$$

where $\theta i$ is the directional angle value of the $i$-th feature point, $d$ is the sampling distance. The starting point's coordinates are initialized to 0 before computing the position of the points after it. After calculated every point's coordinates by (1) and (2), the starting point's coordinates could be figured out by (3) and (4). MinX and MinY are the minimal values of all $\mathrm{x}$ and $\mathrm{y}$ values.

$$
\begin{aligned}
& x_{1}=0-\operatorname{Min} X \\
& y_{1}=0-\operatorname{Min} Y
\end{aligned}
$$

Then the coordinates of points after the starting point are reconstructed by using (1) and (2) again. Fig.3 shows a reconstruction result.

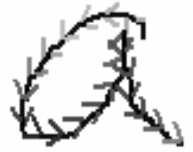

(a)

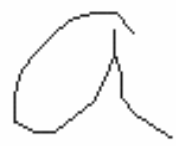

(b)
Fig. 3. (a) The prototype character "a". (b) The reconstructed character from the template

From the reconstructed character, the positional feature points can be sampled along the stroke. By using this reconstruction technique, the size of template files can be reduced without loss of recognition accuracy.

\section{DTW BASED CLASSIFIER DESIGN}

The classifier used in our work is based on prototype matching by DTW. The k-nearest neighbor (k-NN) rule [6] is used as a decision criterion. When performing the directional feature matching by DTW, the local distance is calculated by a quadratic curve equation (5):

$$
d(i, j)=\left\{\begin{array}{cc}
(\Delta \theta)^{2} & 0 \leq \Delta \theta<64 \\
-(\Delta \theta-128)^{2}+8192 & 64 \leq \Delta \theta<128
\end{array}\right.
$$

where $\Delta \theta=\left\{\begin{array}{cc}\left|\theta_{i}-\theta_{j}\right| & 0 \leq\left|\theta_{i}-\theta_{j}\right|<128 \\ 256-\left|\theta_{i}-\theta_{j}\right| & 128 \leq\left|\theta_{i}-\theta_{j}\right|<256\end{array}\right.$,

$d(i, j)$ is the local distance between the $i$-th angle value in template and the $j$-th angle value in input sample, $\theta_{i}$ is the normalized angle value at the $i$-th feature point.

Eq. (5) is based on squared Euclidian distance but with enhanced robustness for complicated characters such as Chinese characters.

Since only directional feature can not present all features of a character, positional feature is combined in our classifier. Some papers described the combination of multi-features when using DTW [3][4]. Some use only positional feature [5]. We use positional feature only for fine classification. After directional feature matching by DTW is performed, all the characters that can be written in a similar stroke order congregated in front of the candidates queue. A generic situation is shown in Fig. 4. From the observation of such generic situation, we employed the following strategy to select the congregated candidates to perform fine classification: 


$$
K=\min (k)
$$

where $\Delta D_{k}>D 1 \times \beta$ or $\Delta D_{k+1} / \Delta D_{k}<\gamma$

$$
G=\left\{C_{i} \mid i \leq K\right\}
$$

where $G$ is the selected set of candidates, $C i$ is the $i$-th candidates, $D_{k}$ is the global distance of the $k$-th candidates, $\Delta D_{k}=D_{k}+1-D_{k} . \beta>1$ and $0<\gamma<1$.

When the set of candidates by (7) is selected, a combined score of each candidate in this set is calculated by:

$$
\text { Stotal }=\text { Sdir }+\alpha \times \text { Spos }
$$

where Sdir is the global directional distance of the candidate, Spos is the global positional distance, $\alpha$ is a constant value chosen by experiments.

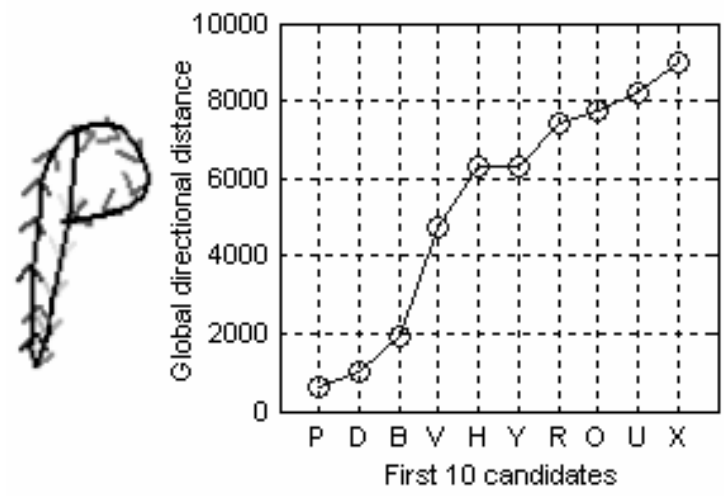

Fig. 4. A handwritten letter "P" and the corresponding curve of the global directional distances of the first 10 candidates.

Each point's coordinates are mapped to a $16 \times 16$ grid by linear normalization before positional feature DTW matching.

All the candidates in the set $G$ are rearranged in ascendant order by Stotal. The first candidate is found as the recognition result.

By using this selecting strategy, the recognition speed can be improved as the positional feature matching is time consuming. Besides, the recognition rate is also found improved in experiments for recognition of uppercase English letters.

\section{EXPERIMENTAL RESULTS}

We collected 75 sets of uppercase English letters by different individuals using our camera user interface. Each set consists of 26 different uppercase English letters. As for lowercase letters, 69 sets collected in the same way are used in our experiments. Some samples among them are illustrated in Fig. 5. All these collected sets are used as test sets.

We use the templates constructed by hand to test the efficiency of English character recognition. The uppercase letter template consists of 151 prototypes; lowercase letter template consists of 258 prototypes. The size of the template file is $3.86 \mathrm{~K}$ and $4.88 \mathrm{~K}$ respectively.

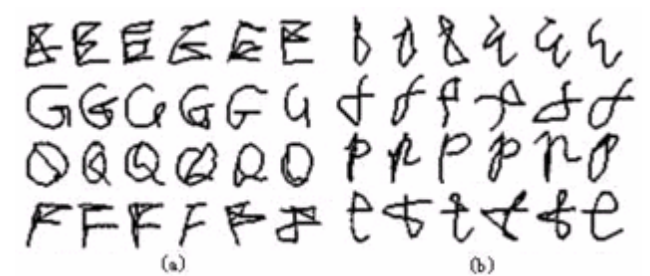

Fig. 5. (a) Some uppercase letter samples collected by our camera user interface; (b) Lowercase letter samples collected by our camera user interface.

In the first experiment, we tested how the precision of angle value affect the recognition results. Experimental results in Table 1 illustrate higher precision angle value can provide higher accuracy.

Table 1. The recognition results of 75 test sets of handwritten uppercase English letters by different precision of directional angle values.

\begin{tabular}{|c|c|c|c|c|c|}
\hline $\begin{array}{c}\text { Number of } \\
\text { normalized } \\
\text { discrete angle } \\
\text { values }\end{array}$ & 16 & 32 & 64 & 128 & 256 \\
\hline $\begin{array}{c}\text { Recognition } \\
\text { rates (\%) }\end{array}$ & 97.3 & 98.1 & 98.6 & 98.7 & $\mathbf{9 8 . 8}$ \\
\hline
\end{tabular}

Another experiment compared different strategy and feature matching. The experimental results are shown in Table 2. It can be seen that directional feature is better than positional feature when recognizing uppercase letters while positional feature is better for lowercase letters. This is because more lowercase letters have similar stroke direction such as "a", "d" and "q", directional feature can hardly classify them. The results also display that the combination of these two features is a success. The recognition rate of lowercase letters raised by more than $10 \%$ after directional and positional feature are combined. Our selecting strategy improved not only recognition speed (raised about 6 times) and also recognition rate for uppercase letters. Some correctly and incorrectly classified uppercase letters are shown in Fig. 6.

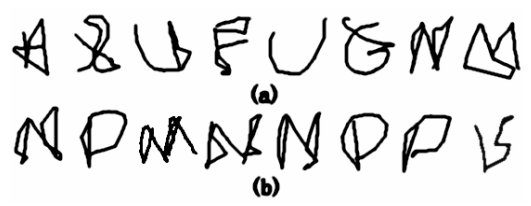

Fig. 6. (a) Some misclassified uppercase letters. (b) Some correctly recognized uppercase letters.

We also compared our modified distance measure by eq. (5) and conventional Euclidian distance when performing directional feature matching. From Table 2 and 3 , the recognition rates raise $0.1 \%$ for uppercase letters and $1.3 \%$ for Chinese characters. This display our local distance measure provides more robustness for complicated characters. 
Table 2. The recognition results of English letters by different strategy and feature matching. $(\mathrm{D}=$ directional feature only; $\mathrm{P}=$ positional feature only; $\mathrm{E}=$ conventional Euclidian distance; $\mathrm{S}=$ modified distance measure by eq. (5); $\mathrm{C}=$ combined both features; $\mathrm{N}=$ not using selecting strategy)

\begin{tabular}{|c|c|c|c|c|c|c|}
\hline $\begin{array}{c}\text { Strategy or } \\
\text { feature used }\end{array}$ & DE & PE & DS & CE & CS & CSN \\
\hline $\begin{array}{c}\text { Recognition } \\
\text { rates of } \\
\text { uppercase } \\
\text { letters }\end{array}$ & 95.0 & 93.0 & 95.1 & 98.7 & $\mathbf{9 8 . 8}$ & 98.4 \\
\hline $\begin{array}{c}\text { Recognition } \\
\text { rates of } \\
\text { lowercase } \\
\text { letters }\end{array}$ & 80.9 & 84.8 & 81.3 & $\mathbf{9 5 . 3}$ & $\mathbf{9 5 . 3}$ & $\mathbf{9 5 . 3}$ \\
\hline
\end{tabular}

Table 3. The recognition rates of 10 sets of handwritten Chinese characters (i.e. $3755 \times 10=37550$ level 1 characters in GB2312-80) by different local distance measure.

\begin{tabular}{|c|c|c|c|c|c|}
\hline \multirow{2}{*}{} & \multicolumn{5}{|c|}{ Cumulative classification rates (\%) } \\
\cline { 2 - 6 } & 1 st & 10 th & 30th & 50th & 100th \\
\hline CE & 70.7 & 85.5 & 89.1 & 90.7 & 92.8 \\
\hline CS & 72.0 & 86.1 & 89.8 & 91.3 & 93.3 \\
\hline
\end{tabular}

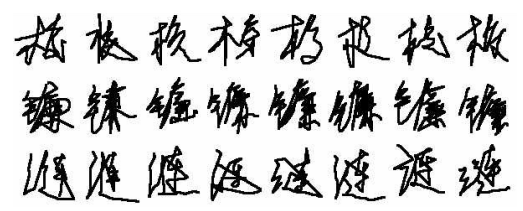

Fig. 7. Some online handwritten Chinese character samples, strokes of each character are connected.

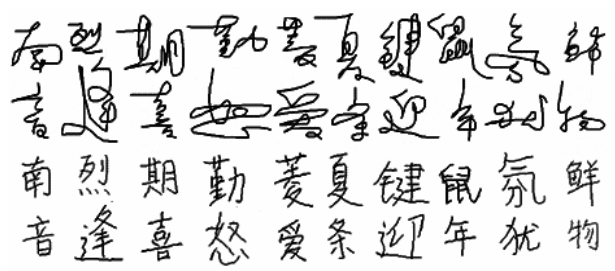

Fig. 8. Some correctly recognized one stroke handwritten Chinese characters and the corresponding prototypes.

The recognition rates in Table 3 are not exciting due to several reasons: all handwritten Chinese characters (testing data and training data) are stroke number and stroke order free but our recognition method is not stroke order free; only one set is used to generate prototypes; we didn't get rid of any error sample in the testing and training data. And all characters are stroke-connected and some testing sets are not well written. Some samples in the test sets are illustrated in Fig. 7. However, our classifier can recognize some terribly distorted characters even some strokes omitted. The cursive style characters in Fig. 8 can be correctly recognized by our classifier and the corresponding prototypes are given below them.

\section{CONCLUSION}

In this paper, we proposed an on-line one stroke cursive character recognition approach which uses a combination method of directional and positional features. Besides, our given local distance measure in directional vector matching by DTW is compared with conventional measure. We also proposed an approach that using only directional vectors to present both the directional and positional features. Thus, the size of templates can be reduced. The experimental results proved the efficiency of our proposed combination method. Although the characters collected by our camera user interface are not well written, the recognition results for such kind of cursive English letters are encouraging. In future work, we are going to employ some off-line recognition method to solve the stroke order problem and improve the efficiency of recognition for one stroke cursive Chinese characters.

\section{ACKNOWLEDGEMENT}

The authors would like to thank Dr. Qiang Huo from the University of Hongkong for providing online handwritten Chinese character samples.

\section{REFERENCES}

[1] R. Plamondon and S. N. Srihari, "On-line and Off-line Handwriting Recognition: A Comprehensive Survey," IEEE Transactions on Pattern Analysis and Machine Intelligence, vol. 22, no. 1, pp. 63-84, January 2000.

[2] C.-L. Liu, S. Jaeger, and M. Nakagawa, "Online Recognition of Chinese Characters: The State-of-the-Art", IEEE Transactions on Pattern Analysis and Machine Intelligence, vol. 26, no. 2, pp. 198-213, February 2004.

[3] Y.-T. Tsay, and W.-H. Tsai, "Attributed String Matching by Split-and-Merge for On-line Chinese Character Recognition", IEEE Transactions on Pattern Analysis and Machine Intelligence, vol. 15, no. 2, pp. 180-185, February 1993.

[4] C. Bahlmann, B. Haasdonk and H. Burkhardt, "On-line Handwriting Recognition with Support Vector Machines - A Kernel Approach," Proc. 8th Int. Workshop Frontiers in Handwriting Recognition, pp. 49-54, August, 2002

[5] V. Vuori et al., "Experiments with Adaptation Strategies for a Prototype-Based Recognition System for Isolated Handwritten Characters," Int. J. Document Analysis and Recognition, vol. 3, no. 3, pp. 150-159, 2001.

[6] E. Fix and J.L. Hodges, "Discriminatory analysis nonparametric discrimination: Consistency properties", Tech. Rep. Number 4, Project Number 21-49-004, USAF School of Aviation Medicine, Randolph Field, Texas, 1951

[7] D. Sankoff and J. B. Kruskal, Time warps, string edits, and macromelecules: the theory and practice of sequence comparison. Addison-Wesley, 1983. 